\title{
1 SexFindR: A computational workflow to identify young and old sex chromosomes
}

4 Phil Grayson $^{1,2^{*}}$, Alison Wright ${ }^{3}$, Colin J. Garroway ${ }^{1}$, Margaret F. Docker ${ }^{1}$

5 1. University of Manitoba, Department of Biological Sciences, Winnipeg, MB, Canada.

6 2. Harvard Medical School, Department of Biomedical Informatics, Boston, MA, USA.

$7 \quad *$ Present address

8 3. Ecology and Evolutionary Biology, School of Biosciences, University of Sheffield, $9 \quad$ Sheffield, UK.

11 Keywords: sex chromosome evolution, sex-linked sequence, population genomics, sea

12 lamprey, sex determination, bioinformatics, k-mer analysis, GWAS, F

13

14 * Corresponding authors: phil.d.grayson@gmail.com, margaret.docker@umanitoba.ca 


\section{Abstract}

18 Sex chromosomes have evolved frequently across the tree of life, and have been a source

19 of fascination for decades due to their unique evolutionary trajectories. They are

20 hypothesised to be important drivers in a broad spectrum of biological processes and are

21 the focus of a rich body of evolutionary theory. Whole-genome sequencing provides

22 exciting opportunities to test these theories through contrasts between independently

23 evolved sex chromosomes across the full spectrum of their evolutionary lifecycles.

24 However, identifying sex chromosomes, particularly nascent ones, is challenging, often

25 requiring specific combinations of methodologies. This is a major barrier to progress in the

26 field and can result in discrepancies between studies that apply different approaches.

27 Currently, no single pipeline exists to integrate data across these methods in a statistical

28 framework to identify sex chromosomes at all ages and levels of sequence divergence. To

29 address this, we present SexFindR, a comprehensive workflow to improve robustness and

30 transparency in identifying sex-linked sequences. We validate our approach using publicly

31 available data from five species that span the continuum of sex chromosome divergence,

32 from homomorphic sex chromosomes with only a single SNP that determines sex, to

33 heteromorphic sex chromosomes with extensive degeneration. Next, we apply SexFindR

34 to our large-scale population genomics dataset for sea lamprey, a jawless vertebrate whose

35 sex determination system remains a mystery despite decades of research. We decisively

36 show that sea lamprey do not harbour sex-linked sequences in their somatic genome,

37 leaving open the possibility that sex is determined environmentally or within the germline 38 genome. 


\section{Introduction}

\section{Sex Chromosome Evolution}

41 Beginning as an identical pair of autosomes, sex chromosomes evolve through the 42 acquisition of a master sex-determining locus (Furman et al. 2020). Over time,

43 recombination suppression can occur between the sex chromosome pair and, in many 44 cases, will proceed along the chromosome length, resulting in highly divergent, 45 heteromorphic sex chromosomes (Charlesworth et al. 2005; Wright et al. 2016; Vicoso 46 2019; Furman et al. 2020), where the heterogametic sex is the sex that carries two different 47 sex chromosomes. However, sex chromosome divergence and degeneration are not 48 inevitable, and numerous species of animals and plants exhibit homomorphic sex 49 chromosomes that are largely identical in size and gene content (Vicoso 2019). Studying 50 sex chromosomes at the early stages of divergence can provide valuable data, making it 51 possible to explore the processes driving the formation of new sex chromosomes.

53 Despite a rich body of theoretical expectations (Palmer et al. 2019; Vicoso 2019; Furman 54 et al. 2020), we are still far from a comprehensive understanding of the forces that catalyse 55 sex chromosome formation and turnover. To robustly test these theories, we require 56 contrasts between independently evolved sex chromosomes across the full spectrum of 57 their evolutionary lifecycle. Recent advances in DNA sequencing and genome assembly, 58 alongside reduction in overall costs has made this increasingly possible through data from 59 numerous non-model organisms. Even so, identifying sex chromosomes from sequence 60 data poses a number of challenges, particularly for nascent sex chromosomes at their early 61 stages of divergence (Palmer et al. 2019). A number of pipelines do exist to identify sex 62 chromosomes from sequence data, including SEX-DETector (Muyle et al. 2016), FindZX 63 (Sigeman, Sinclair, et al. 2021), discoverY (Rangavittal et al. 2019), RADSex (Feron et al. 64 2021) and detsex (Gautier 2014). These pipelines employ a range of different 65 methodologies, contrasting patterns of coverage (e.g., FindZX) or heterozygosity (e.g., 66 FindZX, RADSex) between the sexes or segregation of alleles across a pedigree (e.g., SEX-

67 DETector). These are powerful approaches to identify sex-linked sequences, however, they 68 either require specific types of data, such as pedigree information, or a priori knowledge 69 of the sex chromosome system, or are only able to detect sex chromosomes at certain stages 
70 of divergence. This is because as sex chromosomes diverge, they leave distinct signatures

71 in sequence data which change as divergence proceeds, and so a combination of different

72 methods is necessary to identify sex chromosomes among species (Palmer et al. 2019).

73 However, we currently lack a statistical framework to robustly integrate the results of these

74 different methods to identify a high confidence set of sex-linked sequences and determine

75 if a species exhibits genetic sex determination across the full divergence gradient. This is

76 a major barrier to progress as the use of different approaches can lead to discrepancies

77 between studies as a result of methodological biases (Darolti et al. 2021).

\section{Current Approaches for Sex Chromosome Identification}

80 Heteromorphic sex chromosomes can be identified from genomic data mapped to a

81 reference genome with relative ease, because they exhibit large sex-specific differences in

82 genomic coverage that set them apart from the autosomes. In contrast, homomorphic sex

83 chromosomes are often extremely challenging to identify given that they exhibit few

84 differences from each other in gene and sequence content (Palmer et al. 2019).

85 Additionally, sex chromosomes tend to diverge in a stepwise process, and so homomorphic

86 sex chromosomes are often a mosaic of sex-linked regions of different ages (Wright et al.

87 2017; Furman et al. 2020). As a result, most studies rely on a custom, combined analytical

88 approach to identify homomorphic chromosomes, carrying out a variety of genomic

89 analyses, each of which can provide researchers with extensive lists of candidate sex-linked

90 regions (Palmer et al. 2019). To make this problem more challenging, each method also

91 varies in its power to detect sex-specific sequences along the sex chromosome divergence

92 continuum, and so, lists of candidates often do not overlap in an obvious way. For instance,

93 sex differences in genomic coverage will identify highly divergent sex-linked regions that

94 differ in ploidy between the X and Y, whereas sex differences in SNP density are predicted

95 in younger regions of sex chromosomes which still retain high sequence similarity.

96 Although it is generally understood that certain genomic approaches will be more or less

97 effective at detecting sex chromosomes at varying stages of differentiation (Palmer et al.

98 2019), researchers currently lack a comprehensive workflow that incorporates approaches

99 with the full range of sensitivities to streamline and simplify the unwieldy process of

100 identifying sex-specific sequences in a species of interest. 


\section{Measures of Sex-Specific Differentiation with Coverage Approaches}

103 In highly diverged sex chromosome systems, the X and Y (or Z and W) differ in ploidy,

104 which will result in coverage differences between the sexes when sex-linked reads are

105 mapped to the sex chromosomes in the reference genome (Palmer et al. 2019). The majority

106 of the $\mathrm{X}$ will show approximately one half the sequencing depth in males relative to

107 females, and vice versa for the $\mathrm{Z}$ chromosome, and the $\mathrm{Y}$ or $\mathrm{W}$ chromosomes will exhibit

108 male- or female-limited coverage, respectively. This approach is often used successfully

109 with genomic data from only a single male and single female to identify heteromorphic sex

110 chromosomes (Palmer et al. 2019). Large-scale coverage differences have been used to

111 identify sex chromosomes in a range of species, including insects (Vicoso and Bachtrog

112 2015), reptiles (Vicoso et al. 2013), birds (Sigeman, Strandh, et al. 2021), and plants

113 (Müller et al. 2020).

\section{Measures of Sex-Specific Differentiation with $F_{S T}$}

116 F $_{\text {ST }}$ is an index of allelic fixation in populations, and so, if there are high levels of FST

117 within discrete genomic regions when comparing males to females as separate populations,

118 this would suggest that those regions differ between males and females and, therefore,

119 recombination is absent or reduced (Zhou and Stephens 2012). However, if a sex

120 chromosome or sex-linked region is relatively young, it is possible that sex-specific SNPs

121 would not have time to fix completely within a population, and so would not be detected

122 with this approach. FS has recently been used to aid in the identification of the sex

123 determining regions in multiple stickleback and swordtail fishes (Franchini et al. 2018;

124 Dixon et al. 2019).

126 Measures of Sex-Specific Differentiation with Genome-Wide Association Study (GWAS)

127 GWAS identifies associations between a phenotype and a genotype (Klein et al. 2005).

128 By carrying out a GWAS with sex as the phenotype of interest, it is possible to identify

129 SNPs that are strongly and weakly associated with sex. These SNPs can be fixed, or

130 nearly fixed, in either males or females. GWAS has recently been used to identify the sex 
131 chromosomes in poplars, channel catfish (Ictalurus punctatus) and Atlantic salmon

132 (Salmo salar) (Geraldes et al. 2015; Kijas et al. 2018; Bao et al. 2019).

133

134 Measures of Sex-Specific Differentiation with SNP Density

135 In nascent sex-linked sequences, we would expect to see differences in overall SNP density

136 between males and females due to $\mathrm{Y}$ reads still mapping to the $\mathrm{X}$. This is due to the

137 divergent evolutionary trajectories of the $\mathrm{X}$ and $\mathrm{Y}$ following recombination suppression on

138 the Y (Palmer et al 2019). SNP density can identify regions where male or female-specific

139 SNPs are still segregating within their respective populations. A combined approach using

140 both coverage and SNP density was able to help identify the X chromosome in guppy

141 (Poecilia reticulata) (Wright et al. 2017; Sigeman, Sinclair, et al. 2021) and the switch

142 from an XY to ZW system for white poplar (Populus alba) (Müller et al. 2020).

Measures of Sex-Specific Differentiation with Reference Genome-Free k-mer Analyses

145 K-mers refer to unique sequences of " $\mathrm{k}$ " length, that can be identified in raw sequence data.

146 By identifying and comparing all k-mers within and between samples, all variants,

147 including those that occur in unassembled regions can be identified and subsequently tested

148 for associations with the phenotype of interest. This makes k-mer analyses particularly

149 powerful, especially when working with non-model species, fragmentary genomes of low

150 quality, or with no genome at all. This approach has been used to aid the identification of

151 Y sequences in a range of species (Carvalho and Clark 2013; Akagi et al. 2014; Morris et

152 al. 2018; Böhne et al. 2019). One of the drawbacks of using these methods with population-

153 level data has been the immense computation required to calculate counts of 100s of

154 millions or billions of k-mers across samples, however a new algorithm, kmersGWAS, that

155 has significantly reduced compute time and storage requirements shows promise for

156 increasing accessibility for these methods (Kokot et al. 2017; Voichek and Weigel 2020).

157 Given its broad applicability, kmersGWAS is potentially the most powerful and innovative

158 single method for the identification of sex-specific sequences across the entire divergence

159 continuum using population-level sequencing data. 


\section{The SexFindR Workflow}

163 Given the diversity of sex chromosome systems across an evolutionary continuum that

164 ranges from a single sex-linked SNP to highly degenerate $\mathrm{XY}$, and $\mathrm{ZW}$, systems, it is

165 surprising that there is not currently a comprehensive protocol for researchers to follow in

166 order to determine whether or not a species exhibits genetic sex determination across the

167 full divergence gradient (fig. 1a). We designed SexFindR with this purpose in mind and to

168 improve clarity and transparency across studies in the search for sex chromosomes.

170 There are three steps in SexFindR, each of which provides an opportunity to identify sex-

171 linked sequences in a species of interest (fig. 1b). Step 1: SexFindR employs a coverage-

172 based analysis using DifCover (Smith et al. 2018), requiring only a single male and single

173 female mapped to a reference genome, to identify large regions of chromosomal

174 degradation or differentiation. Step 2: A variety of population genomic analyses are run in

175 order to identify top candidate regions. These analyses include the reference-based SNP

176 density, GWAS, and $F_{\mathrm{ST}}$, as well as the reference-free kmersGWAS. Although these

177 methods have the potential to all point to the same region in the genome, they can also each

178 identify unique regions as top candidates, which makes the robust identification of sex-

179 linked sequences challenging. Step 3: Results from the reference-based methods are

180 converted to $10 \mathrm{~kb}$ windows and combined to increase the user's power to focus on specific

181 genomic regions. Once candidate windows are identified in Step 3, the user can return to

182 GWAS, SNP density, and FsT results to determine if fixed or nearly-fixed differences exist

183 between the sexes in the top candidate windows.

185 To demonstrate the power of SexFindR, we use the workflow to identify sex-linked

186 sequences in five species that cover the entire sex chromosome divergence continuum,

187 from homomorphic sex chromosome systems with a single sex-linked SNP, to degenerated

188 heteromorphic systems (fig. 1a). Given this broad range of applicability within a single

189 protocol, including step-by-step methods and scripts available freely online, SexFindR

190 represents a major improvement in the search for sex-linked sequences in non-model

191 species. By using the method with rigid cut-offs, transparency and reproducibility can be

192 achieved across experiments and research groups. We also apply SexFindR to a large-scale 
193 population genomics dataset we generate to assess the possibility of genetic sex

194 determination in the sea lamprey, a species whose basis for sex determination has remained

195 elusive for decades.

197 The Search for the Mechanism of Sea Lamprey Sex Determination

198 Lampreys are an ancient lineage of jawless fishes comprising more than 40 extant species, 199 approximately 500-600 million years diverged from all jawed vertebrates (Smith et al.

200 2013; Docker et al. 2015). This captivating phylogenetic position has led to increased study 201 in recent years, as they can provide important information about evolution in early 202 vertebrates (York et al. 2019). A longstanding mystery of lamprey biology is how sex is 203 determined in this ancient vertebrate lineage, and much work over the past 50 years has 204 tried to address this in the sea lamprey (Petromyzon marinus) and other lamprey species. 205 Male-biased sex ratios under conditions of high population density or slow growth have 206 led to suggestions of environmental sex determination (ESD) in lampreys (e.g., Torblaa 207 and Westman 1980; Docker and Beamish 1994; Johnson et al. 2017), but evidence for ESD 208 is equivocal (Docker et al. 2019). However, evidence for a genetic component to sex 209 determination is lacking. The sea lamprey karyotype is complex $(n=84)$, with many dot210 like microchromosomes (Potter and Rothwell 1970; Covelo-Soto et al. 2014), and attempts 211 to identify an $\mathrm{X}$ and $\mathrm{Y}$, or $\mathrm{Z}$ and $\mathrm{W}$, through karyological approaches have been 212 unsuccessful (Ishijima et al. 2017). Additionally, Restriction-site Associated DNA 213 sequencing (RAD-seq) was unable to identify any sex-specific sequences in European 214 brook lamprey (Lampetra planeri) (Mateus et al. 2013). However, RAD-seq typically 215 sequences only $\sim 0.1-10 \%$ of the genome, and so, it could easily overlook sex-specific 216 markers or even a relatively large sex-specific region.

218 Given the great difficulty associated with the identification of a sex-linked region or sex 219 chromosome in sea lamprey, we believed that they would be an excellent candidate for use 220 of the SexFindR workflow (fig. 1a). We carried out whole-genome resequencing of over 221120 male and 120 female sea lamprey from blood and fin clips to search for the presence 222 of fixed or nearly-fixed differences between the sexes using our new comprehensive 223 protocol. 


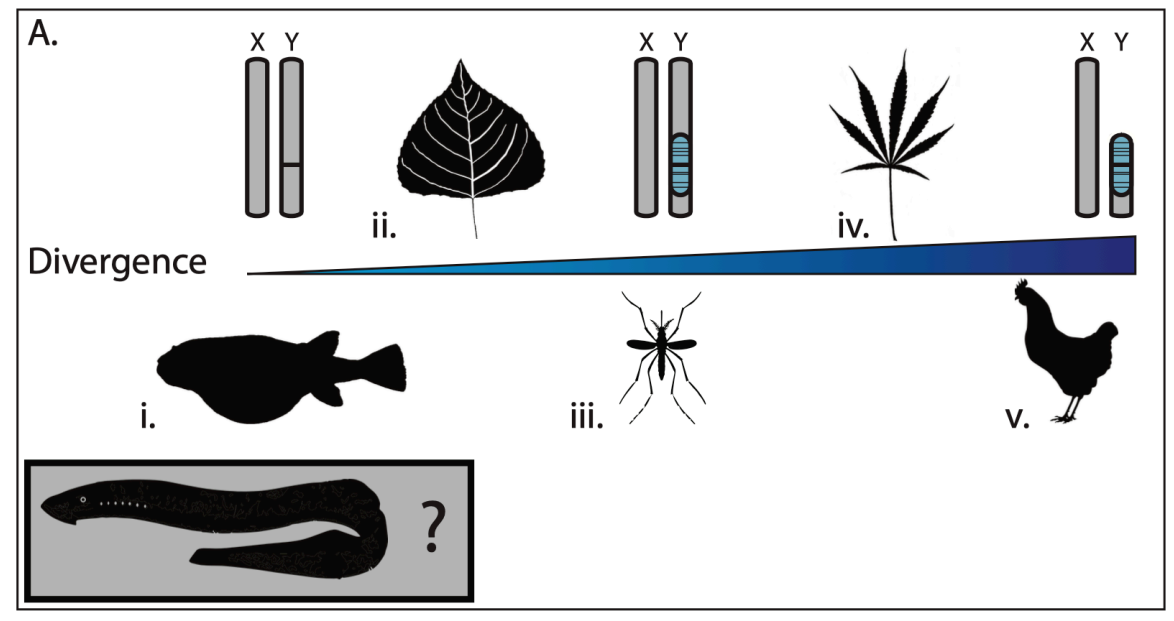

B.

SexFindR Workflow

Step 1. Coverage-based analysis

Single Male

and Female

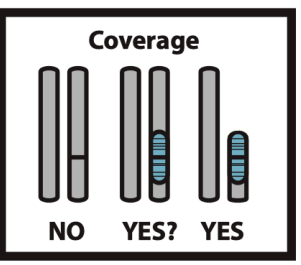

Step 2. Sequenced-based analyses
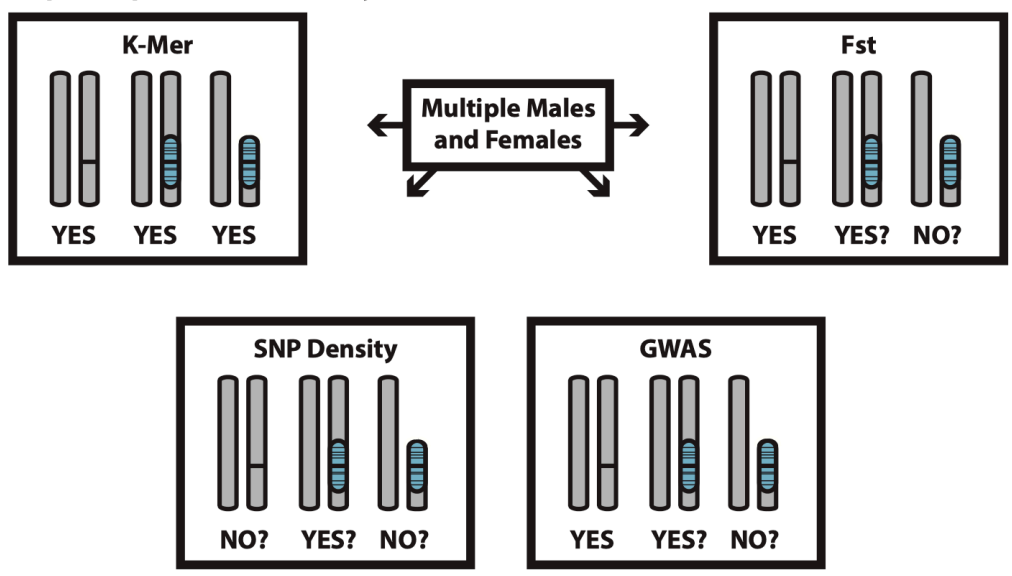

Step 3. Combined sequenced-based analyses
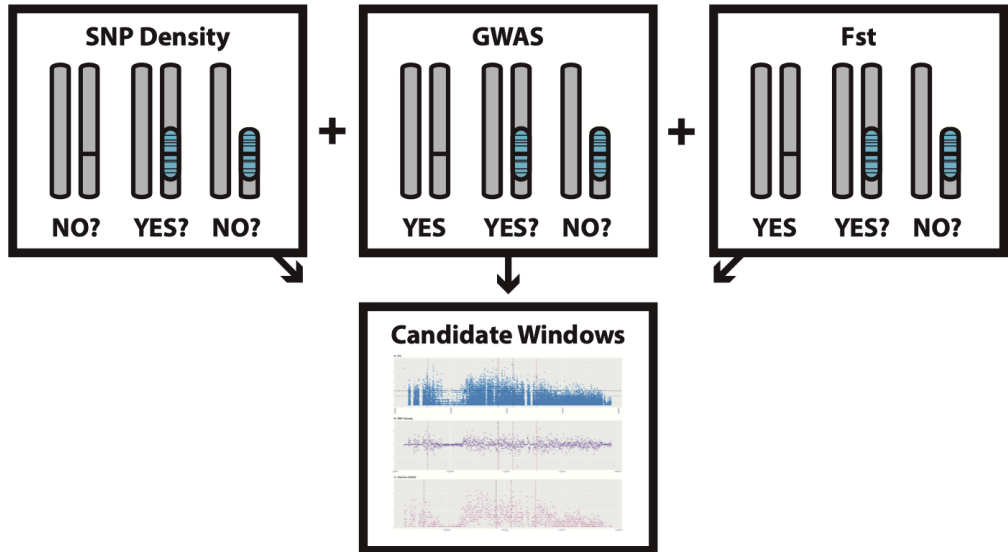
225 Fig. 1. The SexFindR workflow and the sex chromosome divergence continuum. (a)

226 Relative location of five selected species with varying degrees of sex chromosome 227 divergence. i) The tiger pufferfish (Takifugu rubripes) has been previously shown to 228 possess homomorphic XY chromosomes and only a single nucleotide polymorphism 229 (SNP) perfectly linked with sex (Kamiya et al. 2012). ii) Balsam poplar (Populus 230 trichocarpa) has a small, 60-100 kb, Y-like region on the left arm of chromosome 19 in 231 the V2.2 genome (Geraldes et al. 2015; McKown et al. 2017; Müller et al. 2020). iii) The 232 yellow fever mosquito (Aedes aegypti) has homomorphic sex chromosomes with a 233 relatively small $1.3 \mathrm{Mb}$ Y-like "M-locus", but has been shown to have recombination 234 suppression acting across approximately $40 \%$ of the $310.8 \mathrm{Mb}$ chromosome on which it is contained (Fontaine et al. 2017; Aryan et al. 2020). iv) The cannabis plant (Cannabis sativa L) has XY chromosomes that appear nearly identical in size, but has experienced a relatively large amount of XY divergence and a small amount of $Y$-expansion, resulting in a Y chromosome that is $\sim 47 \mathrm{Mb}$ larger than the $\mathrm{X}$ chromosome (Divashuk et al. 2014; Faux et al. 2014). v) Chicken has a ZW system with a $W$ chromosome that has degenerated in a similar manner to the mammalian Y, losing the majority of its ancestral genes (Graves 2006). Inset) The sex determination mechanism in the sea lamprey is currently unknown. (b) The SexFindR workflow in three steps. Each method has a schematic for three positions across the sex chromosome differentiation continuum with a YES/NO below the chromosomes to indicate whether the given method is likely to have power to detect the genomic differences associated with that sex chromosome system. Step 1. Run coveragebased analysis on a single male and female. Step 2. Run variant-based methods (k-mer, SNP density, GWAS, F $F_{S T}$ ) on a population genomics dataset. Step 3. Combine SNP Density, GWAS, and $F_{S T}$ in a window analysis to filter for common hits across referencebased methods. During each step, a user has the potential to identify candidates without the need to continue to additional steps.

251

\section{New Approaches}

253 Here, we present SexFindR, a standardized workflow for the identification of sex-linked 254 sequences along the entire continuum of sex chromosome divergence. SexFindR 255 incorporates the most common genomic approaches used to identify both homomorphic 256 and heteromorphic sex-linked sequences alongside a state-of-the-art reference-free k-mer 257 based analysis. Importantly, SexFindR combines the outputs of these analyses to screen for 258 common candidate regions. We provide SexFindR as a 3-step protocol that allows users to 259 identify sex-specific candidates during any of the 3 steps (fig. 1). To demonstrate the power 260 of SexFindR, we use the workflow to identify the sex-linked sequence in five species that 261 cover the entire divergence continuum, from homomorphic sex chromosome systems with 262 a single sex-linked SNP, to degenerated heteromorphic systems. Finally, we apply 263 SexFindR to our large-scale population genomics dataset to assess the possibility of genetic 
264 sex determination in the sea lamprey. The SexFindR protocol is available at the SexFindR

265 "Read the Docs" page (https://sexfindr.readthedocs.io), and all relevant code can be found

266 on the SexFindR GitHub (https://github.com/phil-grayson/SexFindR).

\section{Results}

269 We designed the SexFindR workflow to allow researchers to robustly identify sex-specific

270 sequences or sex chromosomes in their species of interest. We validated SexFindR in five 271 species with known sex chromosomes that span the divergence continuum and then used 272 it to evaluate the possibility of genetic sex determination in sea lamprey, a jawless 273 vertebrate and invasive threat to commercial and game fisheries throughout the Laurentian 274 Great Lakes in North America.

\section{SexFindR Accurately Validates Known Sex Chromosome Systems}

277 Through the SexFindR workflow, we were able to identify the sex chromosomes in all five 278 positive controls. In Step 1, coverage-based analyses, based on DifCover, robustly 279 identified the known sex chromosomes in chicken, cannabis, and mosquito as outliers using 280 only a single male and single female (fig. 1 and supplementary table S1, Supplementary 281 Material online). This is expected as these specific exhibit heteromorphic, highly diverged 282 sex chromosomes. Specifically, in the chicken, the Z and W chromosomes are annotated 283 in the reference assembly. We find both exhibit significant male-biased and female-biased 284 coverage respectively and are clear outliers relative to the rest of the genome. In cannabis, 285 the $\mathrm{X}$ chromosome has not been identified but $\mathrm{Y}$ sequences are annotated. We were able 286 to identify Y-linked sequences through an obvious shift in coverage relative to the rest of 287 the genome. For mosquito, the chromosome carrying the small 1.3 Mb Y-like "M-locus" 288 was also correctly identified as an outlier (supplementary text 1.1 and fig. S1, 289 Supplementary Material online).

291 For poplar and fugu, coverage analyses in Step 1 were less effective at identifying the 292 known sex chromosomes. While we do find that the XY sex chromosome pair in poplar is 293 enriched for male-biased regions, and is clearly an outlier, individual regions within the 294 chromosome show a less clear pattern. In fugu, no coverage differences are expected 
295 because only one base differs between males and females. Correspondingly, the sex

296 chromosomes are not an outlier regarding sex differences in coverage.

298 We recommend stringent mapping criteria at this step, masking repetitive regions, 299 restricting the number of mismatches when mapping reads to a reference and using only 300 uniquely mapping reads (Palmer et al 2019). This limits incorrectly mapped reads which 301 can mask coverage differences between the sexes and lead to the misclassification of sex302 linked sequences as autosomal.
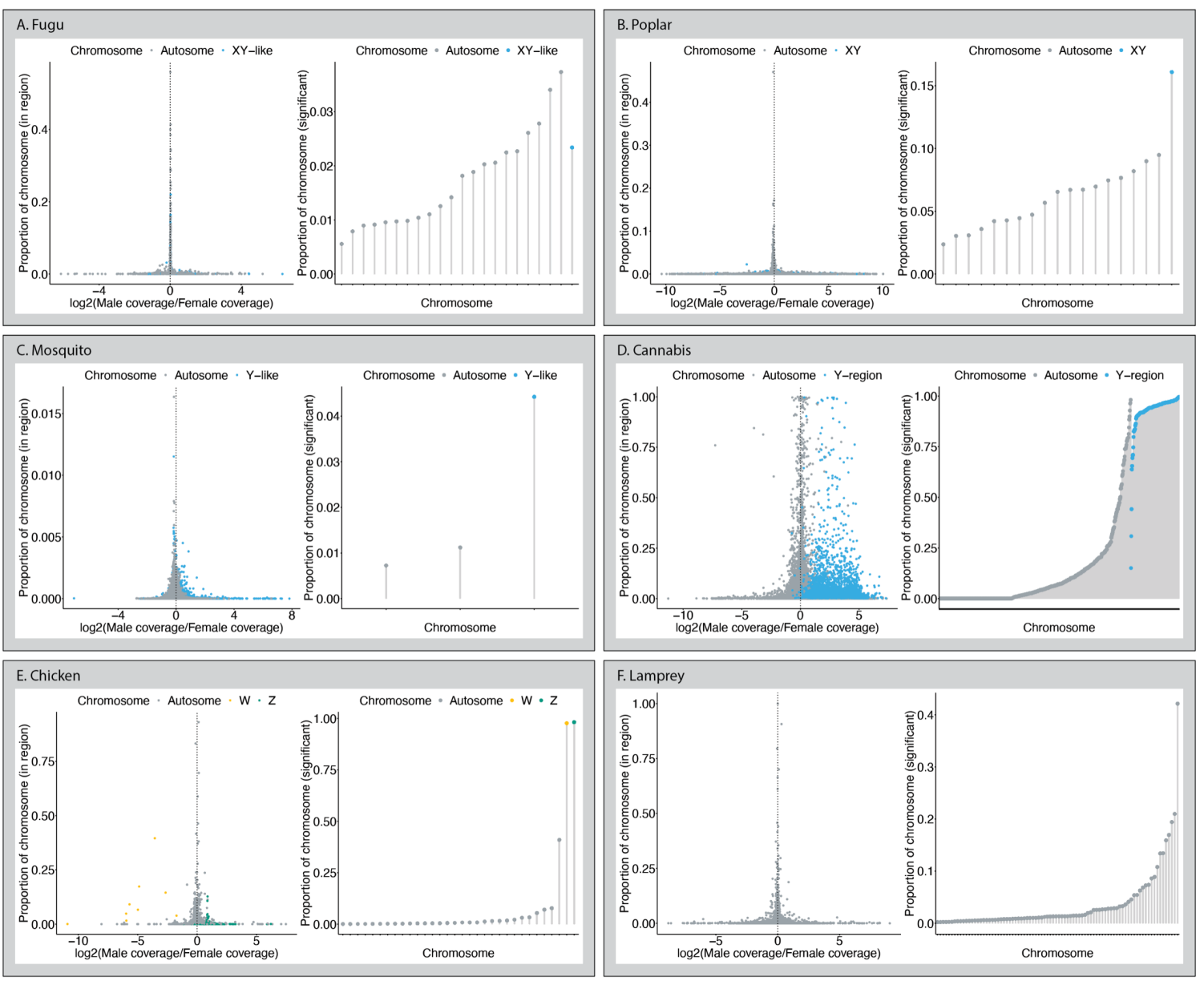

304 Fig. 2. Sex differences in coverage across the genome calculated using DifCover, which breaks chromosomes (or scaffolds) into regions with similar coverage differences between the sexes. For each species (A-F), the left plot has log2(male / female coverage difference) on the $\mathrm{X}$ axis and the proportion of the full chromosome or scaffold that specific region occupies on the $\mathrm{Y}$ axis. The right plot collects all significant regions for each chromosome or scaffold and plots the total proportion of the chromosomal length that displays significantly different coverage between males and females. Known sex chromosomes are 
311 shown in blue (X or $\mathrm{Y})$, orange $(\mathrm{W})$, or green $(\mathrm{Z})$ for each species, except for the lamprey

312 for which has an unknown sex determination system.

313

314 Given that the coverage-based method applied in Step 1 could not robustly identify sex

315 chromosomes in poplar and fugu, we next applied Step 2 of SexFindR to these species. For

316 poplar, all sequence-based methods, including the reference-free k-mer method, were able

317 to positively identify the sex determining region as a top candidate. Importantly, all four

318 methods independently identified the same region as outliers relative to the genome

319 (supplementary text S1.2 and figs. S2 and S4, Supplementary Material online).

320

321 However, for fugu, results from the sequence-based analyses did not perfectly agree,

322 although GWAS and the k-mer-based method were both able to identify the sex 323 determining region as the most significant hits (supplementary text S1.2 and fig. S3, 324 Supplementary Material online). Given the lack of consistent overlap between different 325 approaches, we performed Step 3 of SexFindR on fugu. By combining the non-overlapping 326 signals from SNP density, FST, and GWAS, we were able to filter from over 35,000 10kb 327 windows down to just 5 candidate regions, all of which are located on the previously 328 identified sex chromosome, and one of which contained the sex-determining SNP (fig. 3 329 and supplementary table S2, Supplementary Material online). 


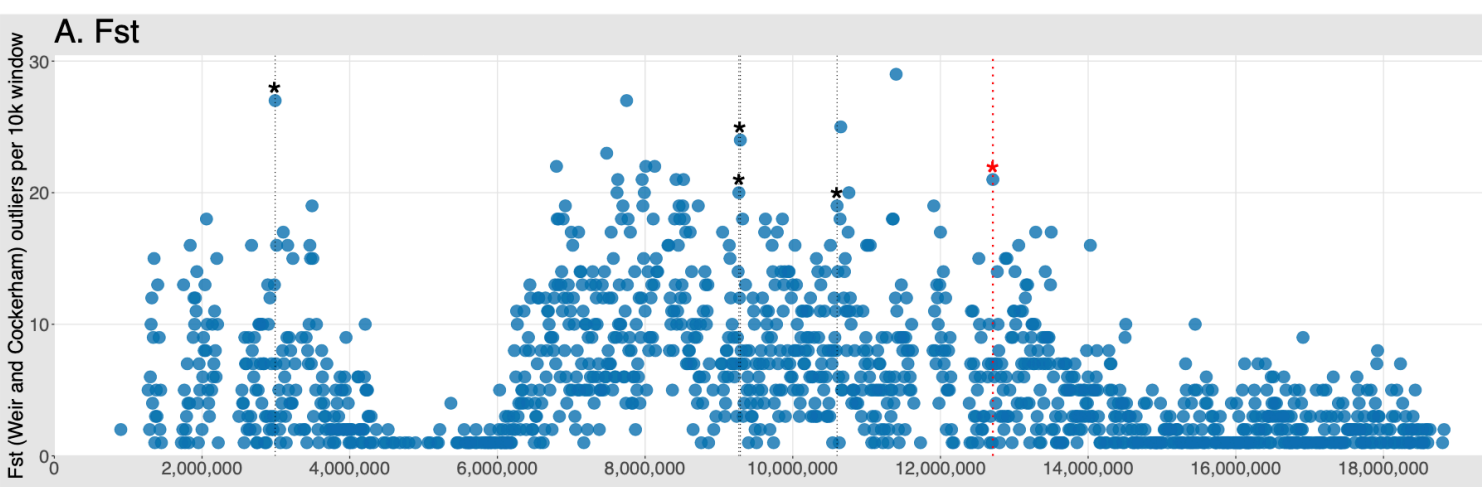

B. SNP Density

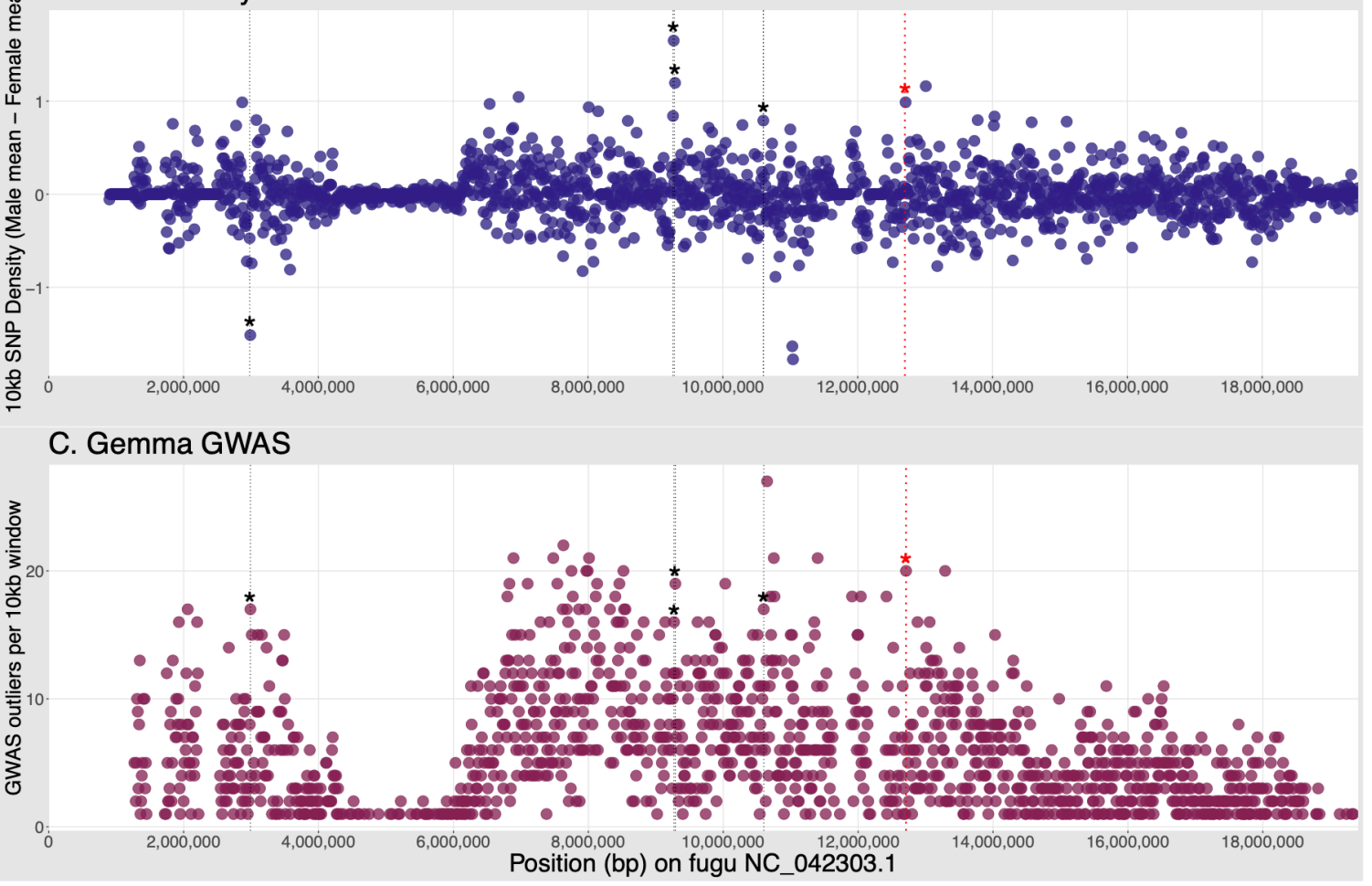

331 Fig. 3. SexFindR Step 3 combined results for $(a) \mathrm{F}_{\mathrm{ST}}$, (b) SNP density, and (c) GWAS identified the fugu sex determining region (red dotted line and red asterisk), alongside

333 four additional candidate windows (thin black dotted lines with black asterisks), all on the 334 known sex chromosome (NC_042303.1).

\section{Sex-linked sequences are not detectable in the sea lamprey somatic}

\section{7 genome}

338 Given our successful identification of all known sex chromosomes in species that span the 339 sex chromosome divergence continuum, from a single SNP to highly degenerate systems, 340 we next applied SexFindR to sea lamprey, a species with an unknown and elusive sex 341 determination system. 
343 First, coverage-based methods in Step 1 did not find consistent male-female enrichment,

344 indicating that lamprey does not have a large region of recombination suppression or a

345 degenerated, or expanded, Y or W chromosome. However, while the lamprey does not 346 have any obvious DifCover regions that exhibit unusual sex differences in coverage (fig.

$3472 f$ left plot and supplementary table S1, Supplementary Materials online), there is a single

348 scaffold that is enriched for sex-biased regions relative to the rest of the genome (fig. $2 f$

349 right plot). This possible sex-linked candidate was disproven with additional analyses, and

350 outliers in the depth analysis appear to be due to large segregating deletions within the sea

351 lamprey (supplementary fig. S7 and text S2.1, Supplementary Materials online).

353 Next, we found no fixed (or nearly-fixed) sequence differences between male and female 354 sea lamprey in Step 2 using SNP density, GWAS, or F F $_{\mathrm{ST}}$, as well as the reference-free 355 kmersGWAS (supplementary text S1.2, Supplementary Materials online). Using the 356 combined approach in Step 3, we did identify a small number of $10 \mathrm{~kb}$ candidate windows 357 (supplementary table S3, Supplementary Materials online), but results were consistently 358 less significant than those from fugu, and when these regions were examined in detail, it 359 was determined that the SNPs driving the signal were segregating in both males and 360 females (supplementary text S1.3, Supplementary Materials online). Notably, we ran 361 SexFindR on lamprey samples collected across a number of different populations. It is 362 possible that the sex determination mechanism or degree of sex chromosome divergence 363 varies across populations, as has been observed in a number of fish, amphibian and reptile 364 species (Furman et al 2020). We tested for this by analysing data from each of the 9 365 populations independently but found no consistent sex-specific SNPs (supplementary text 366 S2.2, Supplementary Materials online). Given that fugu only has a single fixed difference 367 between males and females, and that we were able to identify that site with the SexFindR 368 workflow, this lends support to our conclusion that sea lamprey do not carry sex-linked 369 sequences in their somatic genome. 
372 In this study, we designed a comprehensive workflow, SexFindR, for using next-generation

373 short-read sequencing data to investigate the signals of genetic sex determination in a

374 species of interest. We validate this method in five species with known sex determination

375 systems that span the continuum from a single sex-linked SNP to heteromorphic sex

376 chromosomes. Different approaches to detect sex chromosomes possess varying levels of

377 power along the sex chromosome continuum, and the data and results are often very noisy

378 and difficult to interpret. SexFindR provides a robust and standardised workflow for the

379 community to identify sex-linked sequences. In doing so, we anticipate that this will reduce

380 the potential for discrepancies between studies arising from differences in methodology or

381 parameter choice and facilitate the identification of sex chromosomes in a wide range of

382 non-model species.

383

384 Importantly, we used SexFindR to investigate the possibility of genetic sex determination

385 in the sea lamprey, a jawless fish whose sex determination system has remained elusive for

386 decades. Proving a negative in genomics is quite challenging and publishing a negative

387 result even more so. Without a unified and transparent pipeline, such as SexFindR, it is

388 unclear if sex-linked sequences have not been found for a given species because the right

389 method with the correct parameters has yet to be applied, or if the species truly does not

390 possess sex-linked sequences within the data analyzed. The lack of a consistent or

391 compelling signal of a sex-linked sequence within the sea lamprey genome strongly

392 suggests that there are no fixed (or nearly fixed) sex-specific sequences within our dataset.

393 Although this result does not unequivocally solve the question of sex determination in sea

394 lamprey, it does provide further support for mechanisms other than the standard XY or ZW

395 systems with sex-linked sequence.

397 It is possible that sea lamprey exhibit entirely environmental sex determination.

398 Environmental sex determination has been suggested for some lamprey species, but these 399 data are inconclusive due to the difficulty associated with studying the long and complex 400 lamprey life cycle (Docker et al. 2019). If, on the other hand, sea lamprey do possess a 401 fixed genetic basis for phenotypic sex, it is possible that this region is not present in the 402 somatic sequences. It has been shown that the sea lamprey undergo a programmed genome 
403 rearrangement (PGR) early in the first days of embryonic development (Smith et al. 2009;

404 Smith et al. 2010; Smith et al. 2013; Smith et al. 2018). This PGR results in the deletion of

405 approximately $20 \%$ of the germline genome ( $\sim 500,000 \mathrm{bp})$ from all somatic cells. In sea

406 lamprey, the ovary and testis develop at different times, both years after this PGR, but it is

407 possible that sex-specific germline sequence could be responsible for this differentiation

408 (Docker et al. 2019). Despite our use of a germline reference genome, the samples used in

409 this study all originated from a large population genomics project which collected blood

410 and fin clips, which are somatic tissues; therefore, any sex-specific sequence present only

411 in the germline genome would not be identified within this dataset. Carrying out population

412 genomics using the lamprey germ cells, or examination of the transcriptome or regulatory

413 landscape in developing gonads could shed light on this possibility. In fact, recent work

414 carried out in our laboratory using transcriptomics on developing gonadal tissue from sea

415 lamprey has uncovered a striking signal (Yasmin et al. 2021). By conducting comparative

416 transcriptomic experiments on developing testes and ovaries, Yasmin and colleagues

417 (2021) were able to discover that the 638 genes found within the germline-specific regions

418 exhibit a 36x greater odds of being expressed in testes compared to ovaries, while many of

419 these genes have paralogs in the somatic genome that do not experience differential

420 expression. These male-specific germline genes are likely to be involved in gonadal

421 differentiation and possibly sex determination itself, and include genes known to be

422 important for sex determination in other species.

423

\section{Materials and Methods}

\section{Positive Controls}

\section{SexFindR Step 1. Coverage-Based Analysis}

427 Samples for fugu, poplar, mosquito, cannabis and chicken were downloaded from various

428 sources and mapped to their reference genomes (supplementary table S4, Supplementary

429 Materials online) using Bowtie2 (v 2.3.4.3) (Langmead and Salzberg 2013). Using

430 stringent mapping threshold, such as uniquely mapping reads, is critical to minimise noise

431 and ensure that coverage differences between males and females can be detected (Darolti

432 et al. 2021) BAM files were sorted with Samtools sort and indexed using Samtools index

433 (both v 1.10). 
435 Given that in an XY or ZW system the homogametic sex has two copies of the X or Z, the

436 heterogametic sex, XY males and ZW females, should show approximately one half the

437 sequencing depth for any reads mapping to the $\mathrm{X}$ or $\mathrm{Z}$ respectively. With sequence data

438 from a single male and single female, large regions of sequence divergence or

439 chromosomal degeneration can be identified if present. To test for genomic regions that

440 differ in coverage between males and females, we used DifCover, a program that searches

441 for differences in coverage via an interval-based method that has been used to identify both

442 germline-specific and sex-specific markers (Keinath et al. 2018; Smith et al. 2018;

443 Timoshevskiy et al. 2019). Unlike some window-based approaches, DifCover does not

444 report scores for each window of a predefined length. Instead, it carries out a two-step

445 process: first, given a user-defined length of valid bases (e.g., 1000 bp), DifCover scans

446 each chromosome and scaffold to generate initial windows, where valid bases fall within a

447 range of sequencing depths (e.g., 10x to 240x) in at least one of the samples analyzed. Once

448 average male/female coverage differences have been calculated for these initial windows,

449 these windows are merged based on their similarity to surrounding windows to generate

450 the variably-sized regions we report. The goal with the "valid bases" is to eliminate the

451 signal from problematic regions, including highly repetitive and gapped alignments (Smith

452 et al. 2018).

453

454 Previously, DifCover has been run with a $\log 2$ cut off of 2, which represents a 4-fold 455 change in mapping between the two samples. Given that the expectation for ZW and XY

456 systems should be a change lower than 4-fold, DifCover was run with a lower log2 cut off 457 of 0.740 , which corresponds to a ratio of $1.2 / 2$ or $2 / 1.2$ in case the standard cut off of 4 was 458 too high to identify a simple 2-fold change in coverage expected for the X in XY systems 459 and the Z in ZW systems. This cut off allows us to identify Y-or W-linked reads that show 460 very high levels of enrichment in the male or female respectively and also identify X- or 461 Z-linked reads that show a ratio closer to $1 / 2$ or $2 / 1$ between males and females. All other 462 parameters remained at default settings. 
465 For the positive controls, Step 2 of SexFindR was only run on poplar and fugu, given that

466 Step 1 was able to correctly identify sex chromosomes in mosquito, cannabis and chicken.

467 Population genomic samples for fugu and poplar were downloaded from NCBI

468 (supplementary tables S4-S6, Supplementary Materials online). For reference-based

469 analyses (e.g., F FT, GWAS, and SNP density), reads were mapped to the reference genome

470 using Bowtie2 as above. BAM files were used as input to the Platypus Variant Caller

471 (https://github.com/andyrimmer/Platypus) using joint calling (Rimmer et al. 2014). The

472 resulting VCF was filtered to retain only those sites that had "PASS" under the "FILTER"

473 column using bcftools (v 1.9) (Li et al. 2009).

475 For F $_{\text {ST, }}$, the VCF was also filtered to include only biallelic sites with no missing data. F 476 was calculated, comparing the males and females as separate populations at each SNP 477 using VCFtools (v0.1.14) (Danecek et al. 2011). F ST $_{\text {ST }}$ sites were ranked from highest to 478 lowest $\mathrm{F}_{\mathrm{ST}}$ value to identify top candidates.

480 For GWAS, the VCF was used as input for the program GEMMA (v0.98.1), to identify 481 any SNPs that correlate significantly with phenotypic sex (Zhou and Stephens 2012). 482 VCFtools was used to convert the VCF file to PLINK format while removing indels, 483 requiring at least 50\% coverage of the site, and requiring the minor allele frequency to be 484 between 0.05 and 0.95 . GEMMA was run with default parameters for a binary trait 485 (affected/unaffected), which automatically filters for biallelic sites. Sites were sorted from 486 lowest to highest $p_{-}$lrt to identify top candidates.

488 For SNP density, the VCF file was filtered to include only binary sites and then a single 489 VCF file was created for each individual sample using bcftools. VCFtools snpdensity was 490 used to calculate SNP density for each sample in non-overlapping $10 \mathrm{~kb}$ windows across 491 the genome. The mean SNP density for males and females and the difference between the 492 population means were calculated using an in-house R script (see Read the Docs). In order 493 to determine if these differences were greater than expected by chance, $p$-values were 494 assigned to the difference observed in each window using a permutation test. For each 495 permutation, the sex of all samples was randomized while maintaining the correct number 
496 of males and females in the dataset. An average SNP density for these permutated "males"

497 and "females" was calculated as above. Following the completion of 100,000 permutations,

498 a p-value was calculated for each window in the genome, based on how many permutations

499 were more extreme than the true calculated value (either a greater positive or negative

500 difference between the permuted averages compared to the true average). Windows with $p$

$501<=0.05$ were retained, sorted by p-value, and finally sorted by absolute difference in

502 change between the male and female SNP density means to identify top candidates.

503

504 For kmersGWAS, we used default methodology with slight modifications. For each

505 sample, 31-mers were counted from unmapped fastq data as canonical (count has a 506 minimum of 2), and noncanonical (no minimum count). The two k-mer lists were combined 507 with strand information to generate a single file per sample. The k-mers for all samples 508 were combined using the following criteria: the k-mer has to appear in at least 5 individuals 509 and it must appear in the canonized list for at least $20 \%$ of the individuals that it appears 510 in. A presence/absence count table was generated for samples for every k-mer that passed 511 the filter from the previous step. The k-mer table was converted to PLINK binary format 512 with a minor allele frequency of 0.05 and minor allele count of five. PLINK 1.07 was run 513 with the --noweb, --bfile, and --allow-no-sex flags to generate p-values for every k-mer in 514 the analysis. k-mers were sorted by p-value and the top k-mers were filtered to a top 515 candidate out-file. The top candidate out-file was parsed using plink_to_abyss_kmers.py 516 to pass to ABYSS (Jackman et al. 2017), which assembles the k-mers into contigs 517 (Jackman et al. 2017). Resulting contigs were used as queries for blastn on NCBI to 518 validate the candidates and determine if unassembled sequences were highly significant.

520 SexFindR Step 3. Combined Variant-Based Analysis

521 For the positive controls, Step 3 was only run on fugu, since Step 2 successfully identified 522 the known poplar sex determining region. We developed an in-house linux/R/python 523 workflow to filter for top candidates based on data from FST, SNP density, and GWAS 524 analyses. This produces a single $\mathrm{R}$ data frame where $10 \mathrm{~kb}$ windows are ranked for each 525 analysis to leverage the power of these varied approaches and identify windows that are 526 identified as outliers in multiple analyses. The theoretical inspiration for carrying out this 
527 analysis come from the Composite of Multiple Signals (CMS) program (Grossman et al.

528 2010).

529

530 With SNP density already partitioned into $10 \mathrm{~kb}$ windows and ranked, we selected the top $5315 \%$ of SNPs from the previously-ranked GWAS and F $_{\text {ST }}$ outputs, and counted the number 532 of sites within those same $10 \mathrm{~kb}$ windows for both GWAS and $F_{\text {ST. Windows were then }}$ 533 ranked for each analysis based on the total number of sites contained within. Candidate 534 windows were considered if they ranked within the top 100 for SNP density, GWAS, and 535 FST.

\section{Sea Lamprey}

\section{Sample Collection}

539 Two sets of lamprey samples are used in this study. First, adult sea lamprey $(\mathrm{n}=20$, 540 supplementary table S7, Supplementary Materials online) were collected in June 2019 in 541 the Cheboygan River, MI, USA, a tributary of Lake Huron, during their upstream 542 (spawning) migration. Sterile gloves, pipettes, scalpels, parafilm, and collection dishes 543 were used for each lamprey, and males and females were collected on separate days to 544 control for cross-sample contamination. Blood was collected into an EDTA-coated 545 Vacutainer tube. For the second set of samples, additional whole upstream-migrating sea 546 lamprey $(\mathrm{n}=246$, supplementary table S8, Supplementary Materials online) were collected 547 between May and August 2019 for a large-scale population genomics project that covered 548 the Finger Lakes in New York state, all five Great Lakes (Erie, Huron, Michigan, Ontario, 549 and Superior), and an anadromous population from the Connecticut River (at the Holyoke 550 Dam), MA, USA. Lamprey from the initial Huron samples will be referred to as the 551 "original Huron samples" whenever there is a need to distinguish them from the large 552 population dataset.

554 Abbreviated Protocols for Minimal Animal Involvement, one for the adult sea lamprey 555 collected in June 2019 in the Cheboygan River and one for those collected May-August 5562019 from the Great Lakes, Finger Lakes, and Connecticut River, were approved by the 557 Fort Garry Campus Animal Care Committee at the University of Manitoba. Sea lamprey 
558 were captured at traps during their spawning migration as part of the ongoing sea lamprey

559 control efforts in the Great Lakes basin or collected for other projects, and they were

560 euthanized by Sea Lamprey Control personnel or colleagues from the U.S. Geological

561 Survey according to their approved protocols.

562

563 Sea Lamprey Short-Read DNA Extraction, Sequencing, and Preprocessing

564 For the original Huron samples, DNA was extracted from blood using the DNeasy Blood

$565 \&$ Tissue kit with minor modifications to the standard protocol. $200 \mathrm{uL}$ of blood was used

566 for each individual and the final elution was carried out in $50 \mathrm{uL}$ of Buffer AE. Following

567 quality control and quantification, DNA was shipped on ice to The Center for Applied

568 Genomics (TCAG) where libraries were prepared and sequenced. DNA was first quantified

569 with the Qubit dsDNA HS assay and sheared to a standard 400-bp on the Covaris LE220.

570 Libraries were generated using the Illumina TruSeq PCR-free library preparation protocol

571 with 400-bp insert size on an Agilent Bravo automation system (model B). 700 ng of

572 starting material was used for each library, except for M_9d and M12_d, which only had

$573535 \mathrm{ng}$ and $615 \mathrm{ng}$ of DNA respectively. qPCR was performed on the resulting libraries

574 and they were multiplexed into two pools. Pooll contained 16 samples (low coverage of

$575 \sim 15 \mathrm{x}$ for 8 males and 8 females) and Pool2 contained 4 samples (high coverage of $\sim 60 \mathrm{x}$

576 for 2 males and 2 females). Each pool was sequenced across 3 flowcells of a single Illumina

577 HiSeq X run with 150-bp paired-end reads. Initially, lamprey had been visually sexed at

578 the time of collection. This resulted in one female being mis-labeled as a male (M2_d in

579 all downstream analyses). Following this discovery, all lamprey carcasses were thawed for

580 dissection to visually identify either a testis or ovary. An additional flowcell of Illumina

581 HiSeq X 150-bp paired-end sequencing was carried out for M8_d to replace M2_d as a

582 high coverage male. For the large population samples, DNA was extracted from fin clips

583 taken from sexed frozen carcasses using standard protocol from Qiagen's DNeasy Blood

$584 \&$ Tissue kit with minor modifications. The tissue was allowed to incubate for 18-20 hours

585 for full proteinase $\mathrm{K}$ digestion, and $100 \mathrm{uL}$ of warmed $\left(70^{\circ} \mathrm{C}\right)$ Buffer $\mathrm{AE}$ was allowed to

586 incubate for 1 minute prior to final elution. Following DNA extraction, all samples were

587 once again sent to TCAG for identical library preparation, but the resulting libraries were

588 sequenced on an Illumina NovaSeq S4 flowcell at 2x150 bp. FastQC (v 0.11.8) was run on 
589 all libraries as a quality control measure, and all fastq files performed as expected (Andrews

590 2010). Over-represented sequences and adapters were removed from raw sequencing data

591 using Trimmomatic (v 0.36) (Bolger et al. 2014) prior to second run of FastQC to confirm

592 trimming.

593

594 Sea Lamprey Read Mapping and Variant Calling

595 Following quality control, sea lamprey reads were mapped to the newest germline lamprey 596 genome (kPetMar1 from NCBI

597 https://ftp.ncbi.nlm.nih.gov/genomes/all/GCF/010/993/605/GCF_010993605.1_kPetMar

598 1.pri/GCF_010993605.1_kPetMar1.pri_genomic.fna.gz) using Bowtie2 (v 2.3.4.3) with 599 stringent mapping criteria (-X 1000 --fr --no-mixed --no-discordant) (Langmead and 600 Salzberg 2013). BAM files were sorted with Samtools sort, and indexed using Samtools 601 index (both v 1.10) (Li et al. 2009). Deduplication was carried out using Picard 602 MarkDuplicates (v 2.20.6) and the final BAM files were sorted and indexed as above. The 603 BAM files were used as input to the Platypus Variant Caller 604 (https://github.com/andyrimmer/Platypus) using all samples at once in order to call SNPs 605 jointly (Rimmer et al. 2014). The resulting VCF was filtered to retain only those sites that 606 had "PASS" under the "FILTER" column using bcftools (v 1.9) (Li et al. 2009).

607

608 SexFindR Step 1. Coverage-Based Analysis

609 Following deduplication, a single male and single female lamprey with deep sequencing $610 \sim 60 \mathrm{x}$ were selected for DifCover analysis, which was conducted as above. To further 611 screen the resulting candidate regions, a 2-by-2 DifCover analysis was also carried out 612 (supplemental text 2.1, Supplementary Materials online).

613

614 For lamprey, additional runs of DifCover were conducted to account for our sequencing 615 strategy which included deep sequencing for 2 males and 2 females. Each deeply 616 sequenced female was run against each deeply sequenced male, resulting in 4 experimental 617 comparisons. The two males were run against each other, as were the two females, to serve 618 as controls. Finally, all low-coverage females from the original Huron samples dataset were 619 merged to produce a single file which was compared to a merged file containing all low- 
620 coverage males. Bedtools (v2.25.0) makewindows was used to generate $10 \mathrm{~kb}$ windows

621 across the genome, and bedtools annotate (v2.25.0) was used to map significant regions

622 back to the genome. In R, filtering was carried out to identify candidate regions of overlap

623 in all 4 experimental comparisons that were not found in the control runs. These regions

624 were then compared against the regions identified in the low-coverage DifCover run to

625 search for consistent regions with differential coverage between the males and females.

626

627 SexFindR Step 2. Sequenced-Based Analysis

628 All steps were carried out as described in the Positive Controls section for FST, GWAS, 629 SNP density and kmersGWAS.

630

631 SexFindR Step 3. Combined Sequence-Based Analyses

632 All steps were carried out as described in the Positive Controls section to combine the

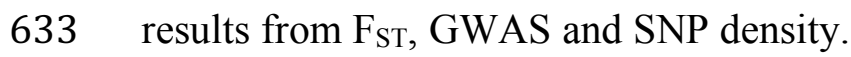

\section{Acknowledgments}

636 Dr. Nick Johnson (US Geological Survey, USGS), Matt Symbal (US Fish and Wildlife, 637 Great Lakes), Gale Bravener (Fisheries and Oceans Canada, Great Lakes), Dr. Ted Castro638 Santos (USGS, Holyoke Dam, Connecticut River), Emily Zollweg-Horan (NY State 639 Department of Environmental Conservation, Cayuga Lake), and Brad Hammers (NY State 640 Department of Environmental Conservation, Seneca Lake) for the sea lamprey samples, 641 and Arfa Khan (University of Manitoba) for performing the DNA extractions.

643 This research was funded by the Great Lakes Fishery Commission Sea Lamprey Research 644 Program (2018_DOC_54073).

645

\section{Author Contribution}

647 MD, CG, AW, and PG conceived the study. PG developed SexFindR, performed the 648 analysis, and wrote the paper with input from all the authors. 


\section{Data Availability}

651 All relevant code can be found at the SexFindR GitHub repo (https://github.com/phil652 grayson/SexFindR) and a detailed walkthrough of the SexFindR protocol can be found at 653 the SexFindR "Read the Docs" page (https://sexfindr.readthedocs.io/en/latest/). Raw 654 sequencing data for sea lamprey will be deposited at NCBI's SRA prior to publication. 


\section{References}

656 Akagi T, Henry IM, Tao R, Comai L. 2014. A y-chromosome-encoded small RNA acts

657 as a sex determinant in persimmons. Science (80- ). 346(6209):646-650.

658 doi:10.1126/science. 1257225.

659 Andrews S. 2010. FastQC: A Quality Control Tool for High Throughput Sequence Data. 660 https://github.com/s-andrews/FastQC.

661 Aryan A, Anderson MAE, Biedler JK, Qi Y, Overcash JM, Naumenko AN, Sharakhova 662 M V., Mao C, Adelman ZN, Tu Z. 2020. Nix alone is sufficient to convert female Aedes 663 aegypti into fertile males and myo-sex is needed for male flight. Proc Natl Acad Sci U S 664 A. 117(30):17702-17709. doi:10.1073/pnas.2001132117.

665 Bao L, Tian C, Liu S, Zhang Y, Elaswad A, Yuan Z, Khalil K, Sun F, Yang Y, Zhou T, et 666 al. 2019. The Y chromosome sequence of the channel catfish suggests novel sex 667 determination mechanisms in teleost fish. BMC Biol. 17(1):1-16. doi:10.1186/s12915668 019-0627-7.

669 Böhne A, Weber AAT, Rajkov J, Rechsteiner M, Riss A, Egger B, Salzburger W. 2019. 670 Repeated evolution versus common ancestry: Sex chromosome evolution in the 671 haplochromine CICHLIDX Pseudocrenilabrus philander. Genome Biol Evol. 11(2):439672 458. doi:10.1093/gbe/evz003.

673 Bolger AM, Lohse M, Usadel B. 2014. Trimmomatic: A flexible trimmer for Illumina 674 sequence data. Bioinformatics. 30(15):2114-2120. doi:10.1093/bioinformatics/btu170.

675 Carvalho AB, Clark AG. 2013. Efficient identification of Y chromosome sequences in 676 the human and Drosophila genomes. Genome Res. 23(11):1894-1907.

677 doi:10.1101/gr.156034.113.

678 Charlesworth D, Charlesworth B, Marais G. 2005. Steps in the evolution of

679 heteromorphic sex chromosomes. Heredity (Edinb). 95(2):118-128.

680 doi:10.1038/sj.hdy.6800697.

681 Covelo-Soto L, Morán P, Pasantes JJ, Pérez-García C. 2014. Cytogenetic evidences of 682 genome rearrangement and differential epigenetic chromatin modification in the sea 683 lamprey (Petromyzon marinus). Genetica. 142(6):545-554. doi:10.1007/s10709-014684 9802-5.

685 Danecek P, Auton A, Abecasis G, Albers CA, Banks E, DePristo MA, Handsaker RE, 686 Lunter G, Marth GT, Sherry ST, et al. 2011. The variant call format and VCFtools. 687 Bioinformatics. 27(15):2156-2158. doi:10.1093/bioinformatics/btr330.

688 Darolti I, Almeida P, Wright AE, Mank JE. 2021. A comparison of methodological 689 approaches to the study of young sex chromosomes: A case study in Poecilia. 
bioRxiv.:2021.11.29.470452. doi:10.1101/2021.11.29.470452.

http://biorxiv.org/content/early/2021/12/01/2021.11.29.470452.abstract.

692 Divashuk MG, Alexandrov OS, Razumova O V., Kirov I V., Karlov GI. 2014. Molecular

693 cytogenetic characterization of the dioecious Cannabis sativa with an XY chromosome

694 sex determination system. PLoS One. 9(1):1-7. doi:10.1371/journal.pone.0085118.

695 Dixon G, Kitano J, Kirkpatrick M. 2019. The Origin of a New Sex Chromosome by

696 Introgression between Two Stickleback Fishes. Mol Biol Evol. 36(1):28-38.

697 doi:10.1093/molbev/msy181.

698 Docker MF, Beamish FWH. 1994. Age, growth, and sex ratio among populations of least 699 brook lamprey, Lampetra aepyptera, larvae: an argument for environmental sex

700 determination. In: Balon EK, Bruton MN, Noakes DLG, editors. Women in ichthyology:

701 an anthology in honour of ET, Ro and Genie. Dordrecht: Springer Netherlands. p. 191-

702 205. https://doi.org/10.1007/978-94-011-0199-8_16.

703 Docker MF, Beamish FWH, Yasmin T, Bryan MB, Khan A. 2019. The Lamprey Gonad.

704 In: Lampreys: Biology, Conservation and Control, Vol. 2. Springer, Dordrecht. p. 1-186.

705 http://dx.doi.org/10.1007/978-94-024-1684-8_1.

706 Docker MF, Hume JB, Clemens BJ. 2015. Introduction: a surfeit of lampreys. In:

707 Lampreys: biology, conservation and control. Springer, Dordrecht. p. 1-34.

708 Faux AM, Berhin A, Dauguet N, Bertin P. 2014. Sex chromosomes and quantitative sex

709 expression in monoecious hemp (Cannabis sativa L.). Euphytica. 196(2):183-197.

710 doi:10.1007/s10681-013-1023-y.

711 Feron R, Pan Q, Wen M, Imarazene B, Jouanno E, Anderson J, Herpin A, Journot L, 712 Parrinello H, Klopp C, et al. 2021. RADSex: A computational workflow to study sex 713 determination using restriction site-associated DNA sequencing data. Mol Ecol Resour. 714 21(5):1715-1731. doi:10.1111/1755-0998.13360.

715 Fontaine A, Filipović I, Fansiri T, Hoffmann AA, Cheng C, Kirkpatrick M, Rašić G, 716 Lambrechts L. 2017. Extensive genetic differentiation between homomorphic sex

717 chromosomes in the mosquito vector, Aedes aegypti. Genome Biol Evol. 9(9):2322718 2335. doi:10.1093/gbe/evx171.

719 Franchini P, Jones JC, Xiong P, Kneitz S, Gompert Z, Warren WC, Walter RB, Meyer A, 720 Schartl M. 2018. Long-term experimental hybridisation results in the evolution of a new 721 sex chromosome in swordtail fish. Nat Commun. 9(1):1-11. doi:10.1038/s41467-018722 07648-2. http://dx.doi.org/10.1038/s41467-018-07648-2.

723 Furman BLS, Metzger DCH, Darolti I, Wright AE, Sandkam BA, Almeida P, Shu JJ, 724 Mank JE, Fraser B. 2020. Sex Chromosome Evolution: So Many Exceptions to the Rules. 725 Genome Biol Evol. 12(6):750-763. doi:10.1093/gbe/evaa081. 
Gautier M. 2014. Using genotyping data to assign markers to their chromosome type and to infer the sex of individuals: a Bayesian model-based classifier. Mol Ecol Resour.

728 14(6):1141-1159. doi:10.1111/1755-0998.12264.

729 Geraldes A, Hefer CA, Capron A, Kolosova N, Martinez-Nuñez F, Soolanayakanahally 730 RY, Stanton B, Guy RD, Mansfield SD, Douglas CJ, et al. 2015. Recent y chromosome 731 divergence despite ancient origin of dioecy in poplars (Populus). Mol Ecol. 24(13):3243732 3256. doi: $10.1111 /$ mec. 13126.

733 Graves JAM. 2006. Sex chromosome specialization and degeneration in mammals. Cell. 734 124(5):901-914. doi:10.1016/j.cell.2006.02.024.

735 Grossman SR, Shylakhter I, Karlsson EK, Byrne EH, Morales S, Frieden G, Hostetter E, 736 Angelino E, Garber M, Zuk O, et al. 2010. A composite of multiple signals distinguishes 737 cuasal variants in regions of positive selection. Science (80- ). 166(February):2008-2011.

738 Ishijima J, Uno Y, Nunome M, Nishida C, Kuraku S, Matsuda Y. 2017. Molecular 739 cytogenetic characterization of chromosome site-specific repetitive sequences in the Arctic lamprey (Lethenteron camtschaticum, Petromyzontidae). DNA Res. 24(1):93-101. doi:10.1093/dnares/dsw053.

Jackman SD, Vandervalk BP, Mohamadi H, Chu J, Yeo S, Hammond SA, Jahesh G, Khan H, Coombe L, Warren RL, et al. 2017. ABySS 2 . 0 : Resource-Efficient Assembly of Large Genomes using a Bloom Filter Effect of Bloom Filter False Positive Rate. Genome Res. 27:768-777. doi:10.1101/gr.214346.116.Freely.

Johnson NS, Swink WD, Brenden TO. 2017. Field study suggests that sex determination in sea lamprey is directly influenced by larval growth rate. Proc R Soc B Biol Sci. 284(1851). doi:10.1098/rspb.2017.0262. Suzuki S, Hosoya S, et al. 2012. A trans-species missense SNP in Amhr2 is associated with sex determination in the tiger Pufferfish, Takifugu rubripes (Fugu). PLoS Genet. 8(7). doi:10.1371/journal.pgen.1002798. 8(1):1-14. doi:10.1038/s41598-018-36209-2. Verbyla K. 2018. Evolution of Sex Determination Loci in Atlantic Salmon. Sci Rep. 8(1):1-11. doi:10.1038/s41598-018-23984-1.

759 Klein RJ, Zeiss C, Chew EY, Tsai JY, Sackler RS, Haynes C, Henning AK, SanGiovanni 760 JP, Mane SM, Mayne ST, et al. 2005. Complement factor H polymorphism in age-related 761 macular degeneration. Science. 308(5720):385-389. doi:10.1126/science.1109557. 
762 Kokot M, Dlugosz M, Deorowicz S. 2017. KMC 3: counting and manipulating k-mer

763 statistics. Bioinformatics. 33(17):2759-2761. doi:10.1093/bioinformatics/btx304.

764 Langmead B, Salzberg S. 2013. Bowtie2. Nat Methods. 9(4):357-359.

765 doi:10.1038/nmeth.1923.Fast.

766 https://www.ncbi.nlm.nih.gov/pmc/articles/PMC3322381/pdf/nihms-366740.pdf.

767 Li H, Handsaker B, Wysoker A, Fennell T, Ruan J, Homer N, Marth G, Abecasis G,

768 Durbin R, 1000 Genome Project Data Processing Subgroup. 2009. The Sequence

769

Alignment Map format and SAMtools. Bioinformatics. 25(16):2078-2079.

770 doi:10.1093/bioinformatics/btp352.

771 Mateus CS, Stange M, Berner D, Roesti M, Quintella BR, Alves MJ, Almeida PR,

772 Salzburger W. 2013. Strong genome-wide divergence between sympatric European river

773 and brook lampreys. Curr Biol. 23(15):R649-R650. doi:10.1016/j.cub.2013.06.026.

774 http://dx.doi.org/10.1016/j.cub.2013.06.026.

775 McKown AD, Klápště J, Guy RD, Soolanayakanahally RY, La Mantia J, Porth I, Skyba

776 O, Unda F, Douglas CJ, El-Kassaby YA, et al. 2017. Sexual homomorphism in dioecious

777 trees: Extensive tests fail to detect sexual dimorphism in Populus. Sci Rep. 7(1):1-14.

778 doi:10.1038/s41598-017-01893-z.

779 Morris J, Darolti I, Bloch NI, Wright AE, Mank JE. 2018. Shared and species-specific

780 patterns of nascent Y chromosome evolution in two guppy species. Genes (Basel).

781 9(5):11-14. doi:10.3390/genes9050238.

782 Müller NA, Kersten B, Leite Montalvão AP, Mähler N, Bernhardsson C, Bräutigam K,

783 Carracedo Lorenzo Z, Hoenicka H, Kumar V, Mader M, et al. 2020. A single gene

784 underlies the dynamic evolution of poplar sex determination. Nat Plants. 6(6):630-637.

785 doi:10.1038/s41477-020-0672-9. http://dx.doi.org/10.1038/s41477-020-0672-9.

786 Muyle A, Käfer J, Zemp N, Mousset S, Picard F, Marais GAB. 2016. Sex-detector: A

787 probabilistic approach to study sex chromosomes in non-model organisms. Genome Biol

788 Evol. 8(8):2530-2543. doi:10.1093/gbe/evw172.

789 Palmer DH, Rogers TF, Dean R, Wright AE. 2019. How to identify sex chromosomes

790 and their turnover. Mol Ecol. 28(21):4709-4724. doi:10.1111/mec.15245.

791 Potter IC, Rothwell B. 1970. The mitotic chromosomes of the lamprey, Petromyzon

792 marinus L. Experientia. 26(4):429-430. doi:10.1007/BF01896930.

793 Rangavittal S, Stopa N, Tomaszkiewicz M, Sahlin K, Makova KD, Medvedev P. 2019.

794 DiscoverY: A classifier for identifying y chromosome sequences in male assemblies.

795 BMC Genomics. 20(1):1-11. doi:10.1186/s12864-019-5996-3.

796 Rimmer A, Phan H, Mathieson I, Iqbal Z, Twigg SRF, Wilkie AOM, Mcvean G, Lunter 
797 G. 2014. Integrating mapping-, assembly- and haplotype-based approaches for calling

798 variants in clinical sequencing applications. Nat Genet. 46(8):912-918.

799 doi:10.1038/ng.3036.

800 Sigeman H, Sinclair B, Hansson B. 2021. FindZX : an automated pipeline for detecting 801 and visualising sex chromosomes using whole-genome sequencing data. :1-22.

802 Sigeman H, Strandh M, Proux-Wéra E, Kutschera VE, Ponnikas S, Zhang H, Lundberg 803 M, Soler L, Bunikis I, Tarka M, et al. 2021. Avian Neo-Sex Chromosomes Reveal 804 Dynamics of Recombination Suppression and W Degeneration. Mol Biol Evol. 805 38(12):5275-5291. doi:10.1093/molbev/msab277.

806 Smith JJ, Antonacci F, Eichler EE, Amemiy CT. 2009. Programmed loss of millions of 807 base pairs from a vertebrate genome. Proc Natl Acad Sci U S A. 106(27):11212-11217. 808 doi:10.1073/pnas.0902358106.

809 Smith JJ, Kuraku S, Holt C, Sauka-Spengler T, Jiang N, Campbell MS, Yandell MD, 810 Manousaki T, Meyer A, Bloom OE, et al. 2013. Sequencing of the sea lamprey 811 (Petromyzon marinus) genome provides insights into vertebrate evolution. Nat Genet. 812 45(4):415-421. doi:10.1038/ng.2568. http://dx.doi.org/10.1038/ng.2568.

813 Smith JJ, Stuart AB, Sauka-Spengler T, Clifton SW, Amemiya CT. 2010. Development 814 and analysis of a germline BAC resource for the sea lamprey, a vertebrate that undergoes 815 substantial chromatin diminution. Chromosoma. 119(4):381-389. doi:10.1007/s00412816 010-0263-z.

817 Smith JJ, Timoshevskaya N, Ye C, Holt C, Keinath MC, Parker HJ, Cook ME, Hess JE, 818 Narum SR, Lamanna F, et al. 2018. The sea lamprey germline genome provides insights 819 into programmed genome rearrangement and vertebrate evolution. Nat Genet. 50(2):270820 277. doi:10.1038/s41588-017-0036-1. http://dx.doi.org/10.1038/s41588-017-0036-1.

821 Timoshevskiy VA, Timoshevskaya NY, Smith JJ. 2019. Germline-Specific Repetitive 822 Elements in Programmatically Eliminated Chromosomes of the Sea Lamprey

823 (Petromyzon marinus). Genes. 10(10). doi:10.3390/genes10100832.

824 Torblaa R, Westman R. 1980. Ecological impacts of lampricide treatments on sea 825 lamprey (Petromyzon marinus) ammocoetes and metamorphosed individuals. Can J Fish 826 Aquat Sci.(37):1835-1850.

827 Vicoso B. 2019. Molecular and evolutionary dynamics of animal sex-chromosome 828 turnover. Nat Ecol Evol. 3(12):1632-1641. doi:10.1038/s41559-019-1050-8.

829 http://dx.doi.org/10.1038/s41559-019-1050-8.

830 Vicoso B, Bachtrog D. 2015. Numerous Transitions of Sex Chromosomes in Diptera. 831 PLoS Biol. 13(4):1-22. doi:10.1371/journal.pbio.1002078. 
832 Vicoso B, Emerson JJ, Zektser Y, Mahajan S, Bachtrog D. 2013. Comparative sex

833 chromosome genomics in snakes: differentiation, evolutionary strata, and lack of global

834 dosage compensation. PLoS Biol. 11(8):e1001643. doi:10.1371/journal.pbio.1001643.

835 Voichek Y, Weigel D. 2020. Identifying genetic variants underlying phenotypic variation

836 in plants without complete genomes. Nat Genet. doi:10.1038/s41588-020-0612-7.

837 http://dx.doi.org/10.1038/s41588-020-0612-7.

838 Wright AE, Darolti I, Bloch NI, Oostra V, Sandkam B, Buechel SD, Kolm N, Breden F, 839 Vicoso B, Mank JE. 2017. Convergent recombination suppression suggests role of sexual 840 selection in guppy sex chromosome formation. Nat Commun. 8:1-10.

841 doi:10.1038/ncomms14251. http://dx.doi.org/10.1038/ncomms14251.

842 Wright AE, Dean R, Zimmer F, Mank JE. 2016. How to make a sex chromosome. Nat 843 Commun. 7(May):1-8. doi:10.1038/ncomms12087.

844 http://dx.doi.org/10.1038/ncomms12087.

845 Yasmin T, Grayson P, Docker MF, Good S V. 2021. The germline-specific region of the 846 sea lamprey genome plays a key role in spermatogenesis. bioRxiv.:2021.09.24.461754.

847 http://biorxiv.org/content/early/2021/09/25/2021.09.24.461754.abstract.

848 York JR, Lee EM, Mccauley DW. 2019. The lamprey as a model vertebrate in

849 evolutionary developmental biology. In: Lampreys: Biology, Conservation and Control.

850 Springer, Dordecht. p. 481-526.

851 Zhou X, Stephens M. 2012. Genome-wide efficient mixed-model analysis for association 852 studies. Nat Genet. 44(7):821-824. doi:10.1038/ng.2310. 